\title{
Preparation of photoluminescent enzymatic nanosensors for glucose sensing
}

Shao-wei Gao ${ }^{\mathrm{a}}$, Hong-shang Peng ${ }^{\mathrm{a}}{ }^{*}$, Xiao-hui Wang ${ }^{\mathrm{a}}$, Fang-tian You ${ }^{\mathrm{a}}$, Feng Teng ${ }^{\mathrm{a}}$, Hong-xia Wang ${ }^{\mathrm{b}}$

\begin{abstract}
A novel photoluminescent glucose nanosensor was facilely prepared by coupling glucose oxidase (GOx) with poly-L-lysine coated oxygen nanosensors via a glutaraldehyde-mediated Schiff-base reaction. The GOx molecules residing on particle surface catalyzed glucose with the expense of oxygen, which was detected by the sensing particle core incorporated with the reference dye coumarin 6 and oxygen probe $\operatorname{Pt}(\mathrm{II})-m e s o-$ tetra(pentafluorophenyl)porphine. The proposed glucose nanosensors $(\sim 150 \mathrm{~nm}$ in hydrodynamic diameter) had a quick response time varied from less than 2 minute to 4 minute. Glucose calibration was performed with ratiometric photoluminescence and time-resolved fluorescence (TRF) respectively, and a series of calibration plots were constructed according to determination time. In comparison, the ratiometric method resulted in wide dynamic range (e.g. 2-10 mM) and high limit of detection ( 1-2 mM), while the TRF mode gave narrow dynamic range (e.g. 1-6 mM) with low detection limit $(\sim 0.1-0.2 \mathrm{mM})$. Finally the enzymatic glucose nanosensors were tested in human serum samples with a TRF microplate reader.
\end{abstract}

Keywords: Glucose; Enzymatic nanosensor; Oxygen; Time-resolved fluorescence; Ratiometric photoluminescence

${ }^{a}$ Key Laboratory of Luminescence and Optical Information, Ministry of Education, Institute of Optoelectronic Technology, Beijing Jiaotong University, Beijing 100044, PR China

${ }^{\mathrm{b}}$ Department of neurology, Zhongguancun Hospital, Beijing 100190, PR China

* Corresponding author.

Tel: 86-10-51683414; Email address: hshpeng@bjtu.edu.cn 


\section{Introduction}

As a primary energy source in most organisms and an important metabolic intermediate, glucose is indispensable to all living creatures. Determination of glucose concentration in fluids plays a big role in biochemistry, clinical chemistry and diagnosis of diabetes particularly. [1,2] Tremendous amount of biosensor for glucose has been developed to this end. [3-6] More recently, a great number of nano-systems have emerged as bioprobes for glucose sensing with extra bonus of improved sensitivity due to the increasing surface area of nanoparticles (NPs) and nanoscale sensibility. [7] Based on electrochemical mechanism, for instance, nanoporous materials (eg. Pt thin film [8] and PtPb networks [9]), nanotubes ( $\mathrm{ZnO}$ arrays [10] and carbon nanotube) [11] and hybrid nanostructures (Pd NPs-carbon nanotube [12] and Cu NPsgraphene [13] ) have been fabricated to assay glucose. Unfortunately, these sensing nanosystems may suffer from interference from electroactive species like urea, ascorbate and urate, or absorptive species, and also are difficult to realize non-invasive detection which is much desirable for in vivo measurement.

Optical glucose nanosensors, in contrast, are capable of non-intrusive measuring while immune from the common interfering species. Several types of nanoprobe have been constructed by using of noble metal NPs, semiconductor quantum dots (QDs) or carbon dots, which are further conjugated with bioactive molecules such as concanavalin A, [14] boronic acid [15-18] and glucose oxidases (GOx). [19-22] With the presence of glucose, the sensing nano-systmes either aggregate to give rise to shifted plasmonic absorption or weakened fluorescence, or biocatalytically generate $\mathrm{H}_{2} \mathrm{O}_{2}$ to quench fluorescence of QDs. It is noticed, however, that the widely used detection approach in bulk enzymatic sensor - oxygen-sensitive fluorescence (from oxygen probe) cooperated with GOx - is rarely adopted to build glucose nanosensors. [23] As far as we are concerned, only one such glucose nanosensor has been reported so far, which was constructed by incorporating GOx and a phosphorescent oxygen indicator sulfo-Ru(dpp) into polyacrylamide nanoparticles. [24] Although the phosphorescent nanosensors are sensitive to glucose, the encapsulation of GOx might retard the catalytic oxidation of glucose.

Previously we had prepared poly-L-lysine (PLL) coated oxygen nanosensors that based on oxygensensitive phosphorescence. [25-27] The nanosensors can facilely conjugate with functional groups such as (3-carboxypropyl) triphenylphosphonium through surface amino groups on PLL shell. [28] It naturally occurs to us that coupling GOx molecules with PLL-coated oxygen nanosensors would result in a fluorescent glucose nanosensor. Herein, a novel glucose nanosensor is proposed in this way, which consists of (i) a PLL-coated sensing particle core doped with the reference dye coumarin 6 and oxygen probe Pt(II)meso-tetra(pentafluorophenyl)porphine and (ii) an outer layer of GOx for glucose. The resultant nanosensors, with a hydrodynamic diameter of $\sim 150 \mathrm{~nm}$, are highly sensitive to glucose in both photoluminescence intensity and lifetime, and respective calibration curves are plotted with different determination times. The functionality of glucose nanosensors are tested in human serum samples by using of a time-resolved fluorescence based plate reader, and reasonable results are derived.

\section{Material and methods}

\subsection{Materials}

Polystyrene (PS), poly-L-lysine (PLL), coumarin 6 (C6) and Pt(II)-meso-tetra(pentafluorophenyl)porphine (PtTFPP) were purchased from Sigma-Aldrich. Dodecyltrimethoxysilane (DTS), glucose oxidase (GOx from 
Aspergillus niger, 100 units/mg) and glutaraldehyde (50\%, GA) were from Jiaxing Sicheng Chemicals Co., Ltd (China), Aladdin (China) and Sinopharm (China), respectively. THF, D-glucose anhydrous (99\%) and mineral oil (pure) were obtained from J\&K (China). All reagents were used as received without further purification. Deionized water (DI) was used in all experiments.

\subsection{Synthesis of PLL-coated photoluminescent oxygen nanosensors (PLL-NPs)}

In a typical synthesis, C6, PtTFPP, PS and DTS were dissolved in THF, in a 2:1:47:50 weight ratio, and at a total concentration of $200 \mathrm{ppm}$. Then, $500 \mu \mathrm{L}$ of mixed solution was rapidly injected into $8 \mathrm{~mL}$ water containing $0.16 \mathrm{mg}$ PLL ( $\mathrm{pH} \mathrm{9,} \mathrm{adjusted} \mathrm{by} \mathrm{ammonium} \mathrm{hydroxide)} \mathrm{under} \mathrm{ultrasonication.} \mathrm{The} \mathrm{solution} \mathrm{was}$ then left standing for $2 \mathrm{~h}$, and afterwards dialyzed against water for $24 \mathrm{~h}$ to remove the organic solvent. Finally, the NPs suspension was centrifugated (16000 r/min, $30 \mathrm{~min}$.) to get rid of surplus PLL and other small molecules, and redispersed into DI water at a concentration of $\sim 29 \mathrm{mg} \cdot \mathrm{L}^{-1}$. The resultant PLL-NPs suspension was stored at $4{ }^{\circ} \mathrm{C}$ for further applications.

\subsection{Synthesis of enzymatic photoluminescent glucose nanosensors (GOx-NPs)}

GOx molecules were conjugated to PLL-NPs through GA-assisted crosslinking. Specifically, 2.4 mg GOx molecules were dissolved in $8 \mathrm{~mL}$ of as-prepared PLL-NPs solution. After thoroughly mixing, $14.4 \mu \mathrm{L}$ of GA solution (diluted by 100 times with water) was added into the mixture, followed by shaking with table concentrator for $2 \mathrm{~h}$ at room temperature to complete the reaction. The solution was centrifugated at 16000 $\mathrm{r} / \mathrm{min}$ for $30 \mathrm{~min}$, and GOx-NPs were collected and subsequently redispersed into $8 \mathrm{~mL}$ water with a concentration of $\sim 232 \mathrm{mg} \cdot \mathrm{L}^{-1}$. The resultant GOx-NPs suspension was stored at $4{ }^{\circ} \mathrm{C}$ for further application.

\subsection{Characterization}

TEM images were obtained with an electron microscope (type Hitachi H-800) at an acceleration voltage of $120 \mathrm{kV}$, using NPs aqueous dispersion placed on the TEM specimen support (cuprum). Zeta potentials and hydrodynamic sizes were determined by photon correlation spectroscopy and by dynamic light scattering (DLS), respectively, using a Zetasizer Nano instrument (Malvern Instruments; Www.malvern.com). UV-visible absorption and steady-state photoluminescent spectra were recorded on a UV-3101PC spectrophotometer (Shimadzu) and an F-4600 fluorescence spectrophotometer (Hitachi), respectively. Time-resolved fluorescence (TRF) measurements were performed on a commercial plate reader (Victor $\times 4$; from Perkin-Elmer).

\subsection{Ratiometric photoluminescence measurements and sensor calibration}

The fluorescence intensity-based calibration was carried out in a standard spectrophotometric cuvette, in which $500 \mu \mathrm{L}$ of air-saturated NPs-GOx solution was placed and sealed by $500 \mu \mathrm{L}$ of mineral oil. To the cuvette bottom below oil layer, $125 \mu \mathrm{L}$ of air-saturated glucose solution (in phosphate buffer saline buffer) with different concentrations were injected, respectively, to reach a final concentration of $0,2,4,6,8,10$ and $12 \mathrm{mM}$. Immediately after the addition of glucose, fluorescence spectra were recorded every $0.5 \mathrm{~min}$ with a scanning speed of $1200 \mathrm{~nm} / \mathrm{min}$ under a 393-nm excitation. Because the photoluminescence intensity ratio was time-evolved, 8 seconds was further added to the registration time of each intensity ratio which considered the scanning speed and spectral range (490 - $650 \mathrm{~nm})$. 


\subsection{Time-resolved fluorescence measurements and sensor calibration}

The lifetime-based calibration was performed in a black 96-well microplate. Normally, 8-12 wells were used for one measurement, each filled with $100 \mu \mathrm{L}$ of GOx-NPs solution containing bovine serum albumin (BSA, $0.07 \mathrm{~g} / \mathrm{mL}$ ). The microplate was initially conditioned for 20 min to equilibrate with air and at constant temperature $\left(37^{\circ} \mathrm{C}\right)$. Then $25 \mu \mathrm{L}$ of air-saturated glucose solution (in phosphate buffer saline buffer) with different concentrations were quickly injected, respectively, to reach a final concentration of $0.5,1,1.5,2$, 2.5, 3, 3.5, 4, 5 and $6 \mathrm{mM}$. After addition of $200 \mu \mathrm{L}$ of mineral oil to prevent ingress of air, fluorescence signal was immediately measured on a commercial plate reader (Victor $\times 4$; from Perkin-Elmer). Rapid lifetime determination of phosphorescent nanosensors was performed based on time-resolved fluorescence (TRF) mode. [27, 29] Specifically, two TRF intensities were recorded after delay times of $30 \mu \mathrm{s}\left(\mathrm{t}_{1}\right)$ and 70 $\mu \mathrm{s}\left(\mathrm{t}_{2}\right)$, each with a gating time of $100 \mu \mathrm{s}$ and using $340 \mathrm{~nm}$ excitation and $642 \mathrm{~nm}$ emission filters. Measured TRF intensity signals were converted into lifetime $(\tau)$ values according to the following equation: $\tau=\left(t_{1}-\right.$ $\left.t_{2}\right) / \ln \left(F_{1} / F_{2}\right)$, where $F_{1}$ and $F_{2}$ correspond to the integrated signals over $100 \mu$ s as obtained after delay times of $t_{1}$ and $t_{2}$, respectively.

\subsection{Human serum test}

GOx-NPs were utilized to detect glucose concentration of human serum samples by means of lifetimebased approach. Before measurements, all samples (obtained from Zhongguancun Hospital, China) were diluted 3 times by phosphorate buffer at $\mathrm{pH}$ 7.0. In a black 96-well microplate, $100 \mu \mathrm{L}$ of GOx-NPs solution was placed inside each well. The microplate was initially conditioned for 20 min to equilibrate with air and at constant temperature $\left(37^{\circ} \mathrm{C}\right)$. Then $25 \mu \mathrm{L}$ of diluted serum sample was quickly injected into the well, followed by addition of $200 \mu \mathrm{L}$ of mineral oil. After 2 minutes, phosphorescence signals were measured on a commercial plate reader according to the procedures of TRF method, and the intensity signals were converted into to lifetime consequently. Finally the concentration of glucose in each serum sample was acquired according to calibration curve with 2 minutes determination time.

\section{Results and discussion}

\subsection{Synthesis and characterization of photoluminescent glucose nanosensor}

Oxygen nanosensors (PLL-NPs) were firstly prepared according to previously reported PLL-assisted reprecipitation-encapsulation method. [26] It was demonstrated that abundant surface amino groups were present on the surface of PLL-NPs. Taking advantage of the GA-assisted Schiff-base reaction, therefore, GOx molecules were facilely conjugated to oxygen naonsensors via the crosslinking between primary amino groups, and enzymatic glucose nanosensors were thus derived (denoted as GOx-NPs). The above fabrication process is schematically illustrated in Fig. 1a, wherein cross section of GOx-NPs is depicted. Basically, the oxygen probe PtTFPP and reference dye C6 are randomly distributed inside the particle core consisting of PS and DTS, and GOx molecules are extended outwards from PLL shell. The sensing capability of GOxNPs arises from the cooperation of GOx with oxygen probe - the former specifically catalyzes the oxidation of glucose into gluconic acid and hydrogen peroxide with the expense of oxygen, whilst the later reports the decrease of dissolved oxygen by means of phosphorescence increase and thereby detects glucose indirectly. Because of conjugation with GOx, the average hydrodynamic diameter of GOx-NPs is considerably enlarged $(\sim 150 \mathrm{~nm})$ comparing that of PLL-NPs $(\sim 75 \mathrm{~nm})$, which is consistent with the TEM results (Fig. 1 
$\mathrm{b}$ and 1c). Accordingly, surface charge of GOx-NPs is also changed from $35.7 \mathrm{mV}$ to $-13.8 \mathrm{mV}$ (inset in Fig. $1 \mathrm{~b}$ and $1 \mathrm{c}$ ), corresponding to the transition of particle surface from positively charged PLL to negatively charged GOx. It needs to point out that some homo-coupled by-products may be produced during the GAassisted coupling reaction. For PLL-NPs, it is difficult for them to form NPs-NPs because of the strong electrostatic repulsion. This can be confirmed by the TEM pictures wherein no dumbbell-typed particle is observed. For GOx-molecules, it may yield GOx-GOx conjugates as they are well dissolved in aqueous solution; but even so, GOx-GOx will be removed from GOx-NPs through high speed centrifugation as described in experimental section.

Conjugation of GOx with PLL-NPs is further characterized by absorption spectra. As displayed in Fig. 2a, the 276-nm absorption band in GOx is attributed to amino acid residues, [30] and the 393-nm absorption in PLL-NPs is to PtTFPP. The two bands are both present in GOx-NPs, demonstrating that GOx molecules are successfully coupled to PLL-NPs. Since GOx-NPs are constructed from PLL-NPs, the two NPs should be similar in fluorescent properties. Fig. $2 b$ shows emission spectra of PLL-NPs and GOx-NPs. It can be seen that the probe phosphorescence of PtTFPP in the two nanosensors, as is expected, both peaks at $650 \mathrm{~nm}$, but the maximum of reference fluorescence of C6 shifts from $\sim 500 \mathrm{~nm}$ (PLL-NPs) to $\sim 530 \mathrm{~nm}$ (GOx-NPs). It is known that GOx has an intrinsic fluorescence ranged from 480 to $580 \mathrm{~nm}$ (peaking at $520 \mathrm{~nm}$ ). [31] In addition, imines (Schiff base) resulted from the crosslinking of GOx with PLL also contribute a little to the broad-band fluorescence. [32] Therefore, the superimposition of the above two emissions and that of C6 can reasonably explain the red-shift of reference fluorescence.

It is known that imines are labile to hydrolysis. Chemical stability of the glucose nanosensors in phosphate buffer saline (PBS) was thus studied by DLS and spectral measurement respectively (see supporting information, Fig. S1). The results show that the hydrodynamic diameter of GOx-NPs is slightly increased after 60 hours' incubation, whilst the photoluminescent intensity ratio is constant except for a trivial blue-shift of the reference fluorescence. Obviously GOx-NPs are stable enough for glucose detection as the assay time is in the order of minutes. Although a few imine linkages do hydrolyze in GOx-NPs, which relax the bounded GOx molecules (i.e. enlarged particle size) and reduce the contribution of imine to reference fluorescence (i.e. blue-shifted green emission), the coupling between GOx and NPs might via many imines thanks to the abundant amine groups on particle surface, which greatly improve the stability of nanosensors.

\subsection{Calibration of NPs-GOx in ratiometric photoluminescence intensity}

Sensitivity of as-prepared photoluminescent glucose nanosensor was firstly tested by using of the ratiometric approach, i.e. simultaneously detecting two signals by a single excitation wavelength. In comparison with single-intensity based sensing that can not give quantitative information unless in cases where the precise concentration of the probes is known, the internal-calibration nature of ratiometric method yields more robust signals. Figure 3a displays typical responsive emission spectra of GOx-NPs exposed to glucose solution $(8 \mathrm{mM})$. It is evident that the probe $650-\mathrm{nm}$ emission increases gradually with the oxidation of glucose, but the reference 530-nm emission keeps rather constant, i.e. the designed photoluminescent nanosensors are sensitive to glucose. Time-evolved emission spectra of GOx-NPs were also measured in response to other concentrations of glucose (see supporting information, Fig. S2). By defining $R$ as the ratio 
of the emission intensity of probe (at $650 \mathrm{~nm}$ ) to that of reference (at $530 \mathrm{~nm}$ ), photoluminescence intensity ratios of GOx-NPs are obtained and plotted against time in Fig. 3b. It can be seen that the evolution of intensity ratio differs a lot according to glucose concentration. The higher the concentration, the shorter the response time for a signal (intensity ratio) change of $90 \%$ (from $\sim 2$ to 4 minutes). This is different from previously reported phosphorescent nano-PEBBLE glucose sensors which have a constant response time. [24] The discrepancy is ascribed to different calibration methods: nano-PEBBLE sensors were calibrated under ambient atmosphere, while GOx-NPs were airproofed by an oil layer. In the latter case, the consumption rate of oxygen is in direct proportion to glucose concentration since the amount of dissolved oxygen is limited.

In terms of calibration plot, a series of calibration curves can be constructed from Fig. $3 \mathrm{~b}$ with different determination time (supporting information, Fig. S3). Short determination time renders calibration plots with low sensitivity, and long time results in ones with narrow detection range. Fig. 4 displays a characteristic calibration plot with $\sim 2.5$ minutes determination time. The $Y$-axis is defined as $R / R_{0}$, where $R_{0}$ and $R$ denote the photoluminescence intensity ratio $\left(I_{650} / I_{530}\right)$ of GOx-NPs without and with the presence of glucose at certain concentrations, respectively. The experimental data are well fitted by a Boltzmann fit according to Eq. 1,

$$
\frac{R}{R_{0}}=A_{2}+\frac{A_{1}-A_{2}}{1+\exp \left(x-x_{0}\right) / d x}
$$

where $A_{1}, A_{2}, x_{0}$ and $d \mathrm{x}$ are empirical parameters. The variable $x$ is related to different glucose concentrations. As can be seen, the dynamic range (range within which $95 \%$ of the total signal change occurs) of the glucose nanosensors is $2-10 \mathrm{mM}$. As for the limit of detection (LOD) determined by linear regression method, it differs substantially according to determination time. For example, LOD is $2.35 \mathrm{mM}$ and $1.18 \mathrm{mM}$, respectively, for determination time of 1 and 4 minutes [supporting information, Fig. S4]. It needs to point out that the sensing particle core of GOx-NPs actually follows an approximate linear behavior against dissolved oxygen. [27] The sigmoidal procession against glucose indicates the enzymatic nature of proposed sensing mechanism. [33]

\subsection{Calibration of NPs-GOx in time-resolved fluorescence (TRF)}

Although ratiometric approach features internal calibration, photobleaching of PtTFPP in GOx-NPs virtually undermines the sensing accuracy. It is noticed that the intensity of 650-nm emission is decreased by $\sim 14 \%$ after 5 minutes' irradiation at $393 \mathrm{~nm}$, while that of C6 is rarely changed (supporting information, Fig. $\mathrm{S} 2$ ). The as-obtained intensity ratio of $I_{650} / I_{530}$ therefore is smaller than the real one, and concentration of glucose might be underestimated as a result. As another robust fluorescence-based detection technique, lifetime-based measurement is usually deemed as a reliable means in that it is free of fluctuation from excitation sources as well as from concentration of probes. In this work, the time-resolved fluorescence microplate reader is employed to investigate lifetime-based response of GOx-NPs to glucose. It is found that the aftereffect of photobleaching on GOx-NPs is rather trivial, and only a $2 \%$ decrease of lifetime is resulted after 8 minutes' measurement (supporting information, Fig. S5). It was reported that fluorescence lifetime of single molecule was severely shortened by photobleaching. The slight reduction of lifetime of GOx-NPs should be thanks to the large amount of PtTFPP molecules per particle, only a tiny fraction of which is photobleached. 
Figure 5a displays lifetime-based response of GOx-NPs against different glucose concentrations. It can be seen that phosphorescence lifetime of GOx-NPs increases gradually with the presence of glucose, corresponding to the diminishment of dissolved oxygen. Similar to the case of photoluminescent intensity ratio, the evolution of lifetime as well as response time is dependent on glucose concentration. Taking advantage of lifetime data with certain determination time, various calibration plots can be established (supporting information, Fig.S6). But only calibration curves with determination time longer than 2 minutes are of practical application, considering the detection sensitivity and dynamic range. Figure 5b displays a characteristic calibration plot with 2 min determination time, which can be well fitted by a Boltzmann function described in Eq. 1. The dynamic range is around 1- $6 \mathrm{mM}$, which is narrower than that obtained from intensity-based calibration. Also, the lifetime-derived LOD varies with determination time. It is 0.07 $\mathrm{mM}$ and $0.2 \mathrm{mM}$ for determination time of 2 and 4 minutes respectively (supporting information, Fig. S7). The value is much lower than that from intensity-based approach, indicating that TRF mode can provide more sensitive detection of glucose.

\subsection{Analysis of human serum sample}

As a demonstration of the sensing capability, GOx-NPs were tested glucose in human serum samples. The ratiometric photoluminescence approach was adopted firstly. Unfortunately, the serum samples have a strong autofluorescence that superposes with the reference emission of GOx-NPs (peaking at $513 \mathrm{~nm}$ ), which considerably distorts the ratiometric signals (supporting information, Fig. S8). In comparison, TRF mode is much suitable for determination of glucose in serum samples, as the 30- $\mu$ s delay can effectively eliminate background fluorescence whose lifetime is usually in the order of nanoseconds. Table 1 shows the as-obtained result of three human serum samples, in which the calibration curve in Fig. $5 \mathrm{~b}$ is employed to convert measured lifetime data into glucose concentration. The errors (between $-10.3 \%$ and $-2.9 \%$ ) are acceptable considering the complicated bio-components in serum, which may be unspecifically absorbed onto the surface of GOx-NPs and thereby hamper the enzyme catalytic reaction. Therefore, further modification such as PEGylation is needed for GOx-NPs to work well in bio-environments, and related work is being carried out.

\section{Conclusions}

In summary, photoluminescent enzymatic glucose nanosensors, GOx-NPs, are prepared by coupling GOx with PLL-coated nanoparticles doped with the reference dye C6 and probe PtTFPP through GA-assisted Schiff-base reaction. The GOx molecules reside on particle surface to catalyze glucose with the expense of oxygen, which is reported by the sensing particle core. Because of nanosized dimension (150 nm) and enzymatic nature, GOx-NPs are very sensitive to glucose with a quick response time of a few minutes. Two robust approaches, ratiometric photoluminescence and time-resolved fluorescence, are adopted for glucose calibration, and various calibration curves are resulted according to different determination time. Comparatively, the former technique gives rise to wide dynamic range (e.g. 2-10 mM) and high LOD values (about 1-2 mM), while the latter produces narrow dynamic range (e.g. 1-6 mM) with low LOD values (about 0.1-0.2 mM). In addition, glucose in serum was detected with GOx-NPs by means of TRF mode and reliable results are derived. With further surface biomodificaiton, the glucose nanosensors are promising for in vivo sensing of glucose. 


\section{References}

[1] J.C. Pickup, Z.L. Zhi, F. Khan, T. Saxl, D.J.S. Birch, Diabetes-Metab Res, 24 (2008) 604-610.

[2] J.C. Pickup, F. Hussain, N.D. Evans, N. Sachedina, Biosens Bioelectron, 20 (2005) 1897-1902.

[3] C. Chen, Q.J. Xie, D.W. Yang, H.L. Xiao, Y.C. Fu, Y.M. Tan, S.Z. Yao, Rsc Adv, 3 (2013) 4473-4491.

[4] G.F. Wang, X.P. He, L.L. Wang, A.X. Gu, Y. Huang, B. Fang, B.Y. Geng, X.J. Zhang, Microchim Acta, 180 (2013) 161-186.

[5] R.J. McNichols, G.L. Cote, J Biomed Opt, 5 (2000) 5-16.

[6] M.S. Steiner, A. Duerkop, O.S. Wolfbeis, Chem Soc Rev, 40 (2011) 4805-4839.

[7] K.J. Cash, H.A. Clark, Trends Mol Med, 16 (2010) 584-593.

[8] S. Joo, S. Park, T.D. Chung, H.C. Kim, Anal Sci, 23 (2007) 277-281.

[9] J. Wang, D.F. Thomas, A. Chen, Anal Chem, 80 (2008) 997-1004.

[10] K. Yang, G.W. She, H. Wang, X.M. Ou, X.H. Zhang, C.S. Lee, S.T. Lee, J Phys Chem C, 113 (2009) 20169-20172.

[11] L.N. Cella, W. Chen, N.V. Myung, A. Mulchandani, J Am Chem Soc, 132 (2010) 5024-+.

[12] L. Meng, J. Jin, G.X. Yang, T.H. Lu, H. Zhang, C.X. Cai, Anal Chem, 81 (2009) 7271-7280.

[13] Z.H. Wang, J.F. Xia, X.L. Qiang, Y.Z. Xia, G.Y. Shi, F.F. Zhang, G.T. Han, L.H. Xia, J. Tang, Int J Electrochem Sc, 8 (2013) 6941-6950.

[14] K. Aslan, J.R. Lakowicz, C.D. Geddes, Anal Biochem, 330 (2004) 145-155.

[15] D.B. Cordes, S. Gamsey, B. Singaram, Angew Chem Int Edit, 45 (2006) 3829-3832.

[16] R. Freeman, L. Bahshi, T. Finder, R. Gill, I. Willner, Chem Commun, (2009) 764-766.

[17] W.T. Wu, T. Zhou, A. Berliner, P. Banerjee, S.Q. Zhou, Angew Chem Int Edit, 49 (2010) 6554-6558.

[18] P.F. Shen, Y.S. Xia, Anal Chem, 86 (2014) 5323-5329.

[19] R. Gill, L. Bahshi, R. Freeman, I. Willner, Angew Chem Int Edit, 47 (2008) 1676-1679.

[20] P. Wu, Y. He, H.F. Wang, X.P. Yan, Anal Chem, 82 (2010) 1427-1433.

[21] C. Radhakumary, K. Sreenivasan, Anal Chem, 83 (2011) 2829-2833.

[22] L. Li, F.F. Gao, J. Ye, Z.Z. Chen, Q.L. Li, W. Gao, L.F. Ji, R.R. Zhang, B. Tang, Anal Chem, 85 (2013) 9721-9727.

[23] D.B. Papkovsky, R.I. Dmitriev, Chem Soc Rev, 42 (2013) 8700-8732.

[24] H. Xu, J.W. Aylott, R. Kopelman, Analyst, 127 (2002) 1471-1477.

[25] X.H. Wang, H.S. Peng, Z. Chang, L.L. Hou, F.T. You, F. Teng, H.W. Song, B. Dong, Microchim Acta, 178 (2012) 147-152.

[26] X.H. Wang, H.S. Peng, H. Ding, F.T. You, S.H. Huang, F. Teng, B. Dong, H.W. Song, J Mater Chem, 22 (2012) 16066-16071.

[27] X.H. Wang, H.S. Peng, L. Yang, F.T. You, F. Teng, L.L. Hou, O.S. Wolfbeis, Angew Chem Int Edit, 53 (2014) 12471-12475.

[28] X.H. Wang, H.S. Peng, L. Yang, F.T. You, F. Teng, A.W. Tang, F.J. Zhang, X.H. Li, J Mater Chem B, 1 (2013) 5143-5152.

[29] R.I. Dmitriev, A.V. Zhdanov, G. Jasionek, D.B. Papkovsky, Anal Chem, 84 (2012) 2930-2938.

[30] Q. Zhang, S.Y. Wu, M.W. He, L. Zhang, Y. Liu, J.H. Li, X.M. Song, Acta Chim Sinica, 70 (2012) 2213-2219.

[31] A. Lepore, M. Portaccio, E. De Tommasi, P. De Luca, U. Bencivenga, P. Maiuri, D.G. Mita, J Mol Catal B-Enzym, 31 (2004) 151-158. 
[32] J.M. Urreaga, M.U. de la Orden, Carbohyd Polym, 69 (2007) 14-19.

[33] P.C. Pandey, S. Upadhyay, H.C. Pathak, Sensors and Actuators B: Chemical 60 (1999) 83-89.

\section{Biographies}

Shao-wei Gao is a graduate student of Beijing Jiaotong University in China. She obtains her bachelor's degree from

Jiaotong

research

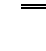

Experiment data

Sample

(mM)
Standard data

(mM)
Relative error

(\%)

Beijing

fluorescent

nanosensors.

\begin{tabular}{llll} 
& & & \\
\hline 1 & 4.08 & -10.3
\end{tabular}

\begin{tabular}{|c|c|c|c|c|c|}
\hline Hong-shang & 2 & 5.94 & 6.36 & -6.6 & Peng received \\
\hline $\begin{array}{l}\text { his } \mathrm{PhD} \text { degree } \\
\text { Jiaotong }\end{array}$ & 3 & 5.67 & 5.82 & -2.9 & $\begin{array}{l}\text { from Beijing } \\
\text { University in }\end{array}$ \\
\hline
\end{tabular}

of Beijing Jiaotong University. His current research interests include fluorescent nanosensors for oxygen and temperature, and fluorescent bioimaging.

Xiao-hui Wang received her PhD degree from Beijing Jiaotong University in 2015 and now is a Postdoc at Beijing University of Posts and Telecommunications. Her research focuses on fluorescent oxygen nanosensor.

Fang-tian You received her $\mathrm{PhD}$ degree from Beijing University in 2002 and now is a professor of Beijing Jiaotong University. Her research focuses on luminescent lanthanide doped materials.

Feng Teng received his $\mathrm{PhD}$ degree from Chinese Academy of Science in 1998 and now is a professor at Beijing Jiaotong Univeristy. His research interests include optoelectronic materials and device.

Hong-xia Wang received her Master degree from Shanxi Medical University in 2005 and now is an associated chief physician at Zhongguancun Hospital, Beijing.

\section{Acknowledgements}

This work was financially supported by the NSFC (Grants 61078069 and 11274038), the NSF for Distinguished Young Scholars (61125505) and the NCET (12-0771).

The authors declare that there is no conflict of interests regarding the publication of this article.

Table 1. Determination of glucose in human serum samples based on TRF mode

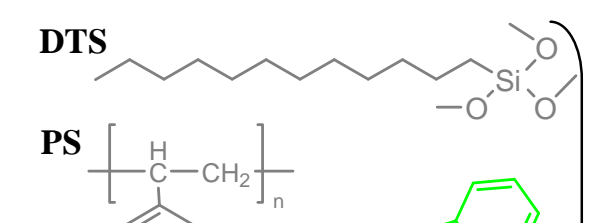




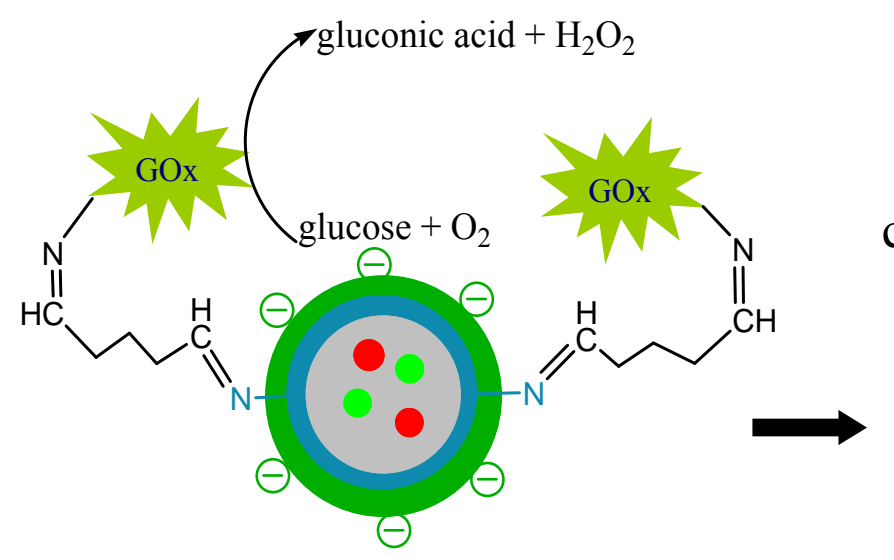

Stage 2 (GOx-NPs)
Stage 1 (PLL-NPs)

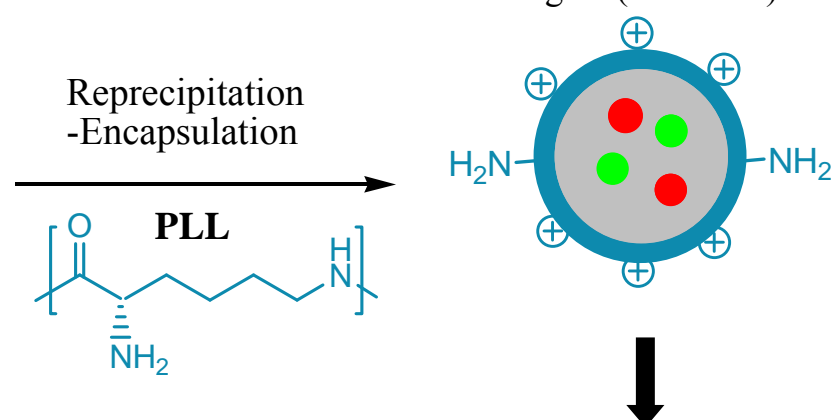

b)
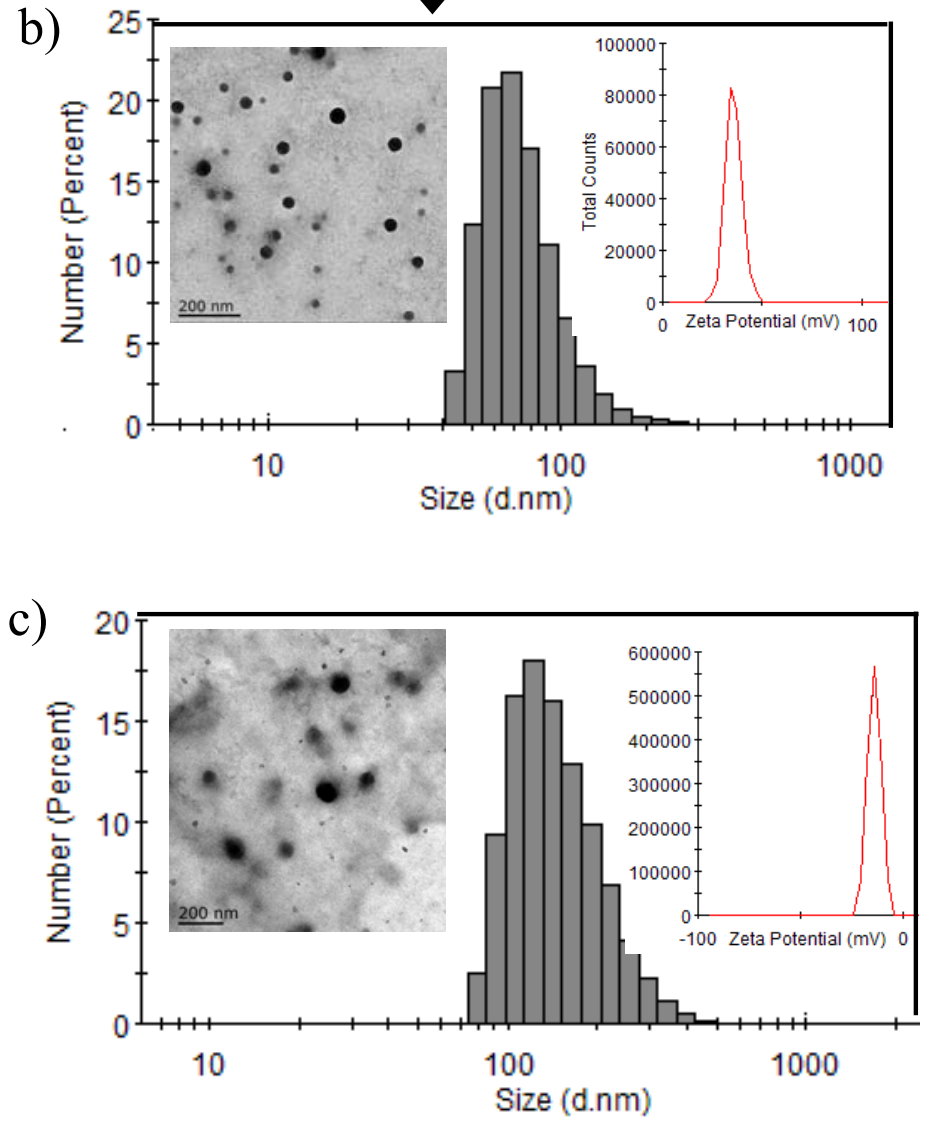

Figure 1. (a) Schematic representation of the synthesis of photoluminescent glucose nanosensor GOx-NPs. From stage 1 to stage 2, PLL-NPs and GOx-NPs are sequentially prepared. (b) Characterizations of PLLNPs and (c) GOx-NPs. DLS and the insets of TEM image and zeta potential, for respective NPs, are provided. 

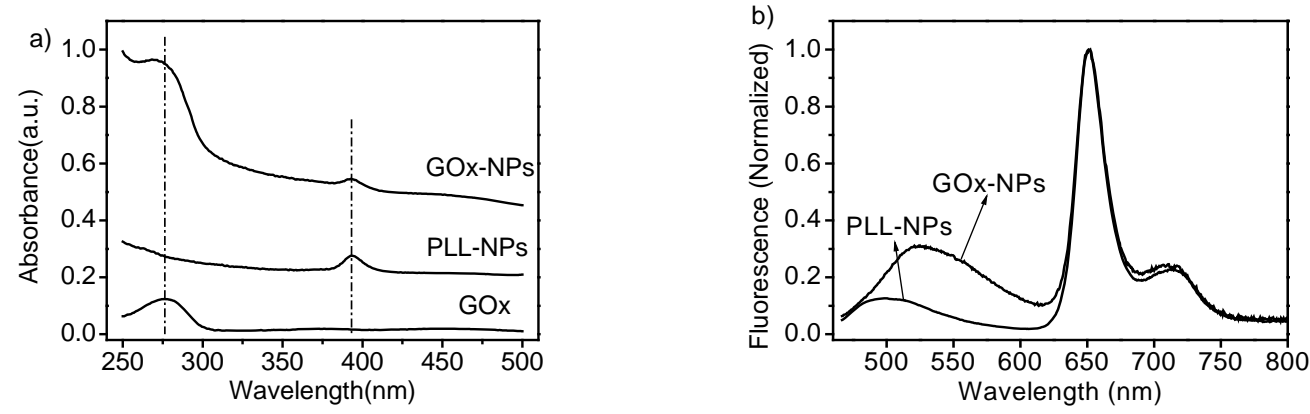

Figure 2. (a) Absorption spectra of GOx, PLL-NPs and GOx-NPs. (b) Emission spectra of PLL-NPs and GOx-NPs. The excitation wavelength is at $393 \mathrm{~nm}$. 

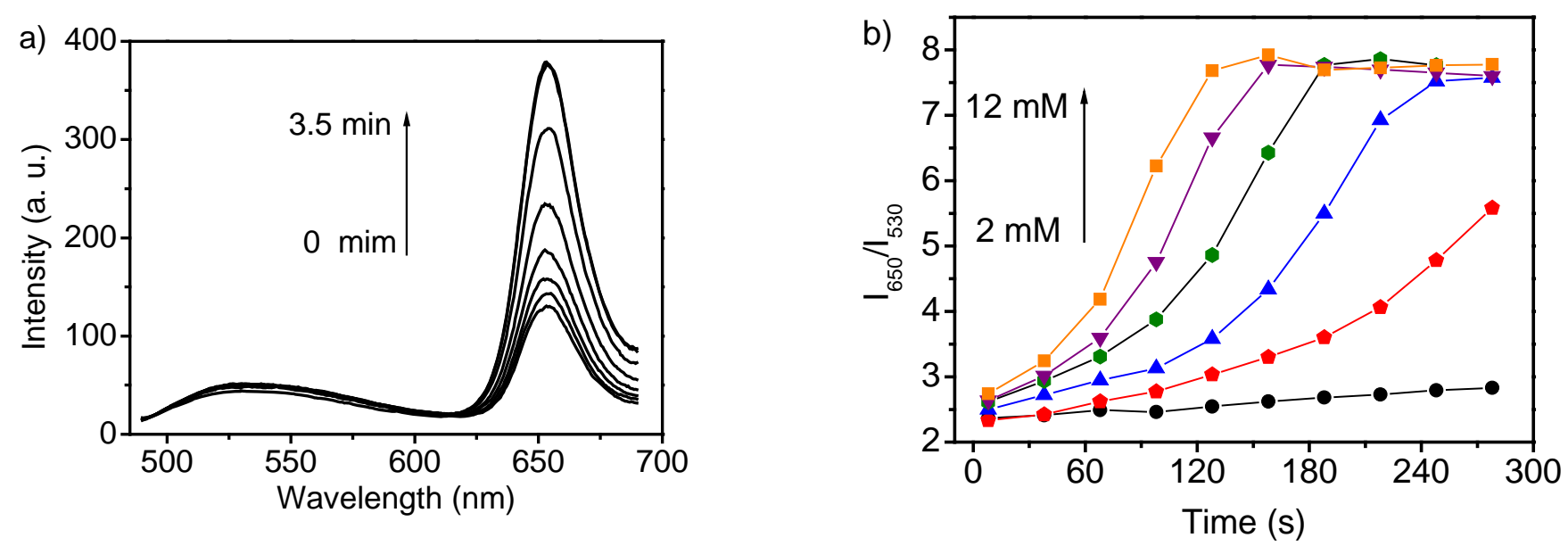

Figure 3. (a) Time-evolved emission spectra of GOx-NPs in response to glucose $(8 \mathrm{mM})$. The spectra are recorded after glucose addition with a time interval of 0.5 min sequentially (room temperature), and the excitation wavelength is at $393 \mathrm{~nm}$. (b) Kinetic response of GOx-NPs to increasing concentration of glucose in intensity ratio $\left(\mathrm{I}_{650} / \mathrm{I}_{530}\right)$. The intensity ratios start at the time of 8 second and are compensated for photobleaching through controlled experiment. The concentration of glucose ranges from 2 to $4,6,8$, 10 and $12 \mathrm{mM}$. 


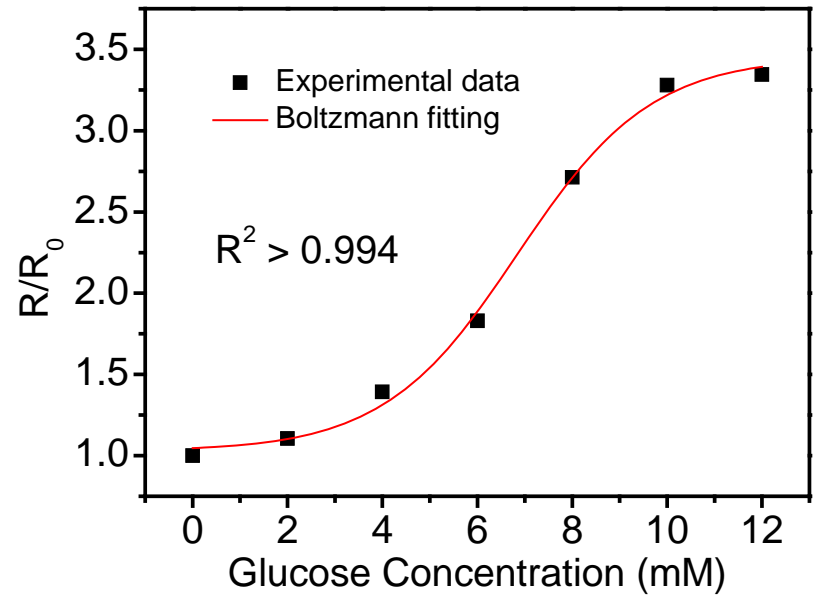

Figure 4. Ratiometric photoluminescence intensity-based calibration plot for GOxNPs. The data are obtained from Fig. 3B with determination time of $158 \mathrm{~s}(\sim 2.5$ minutes). 

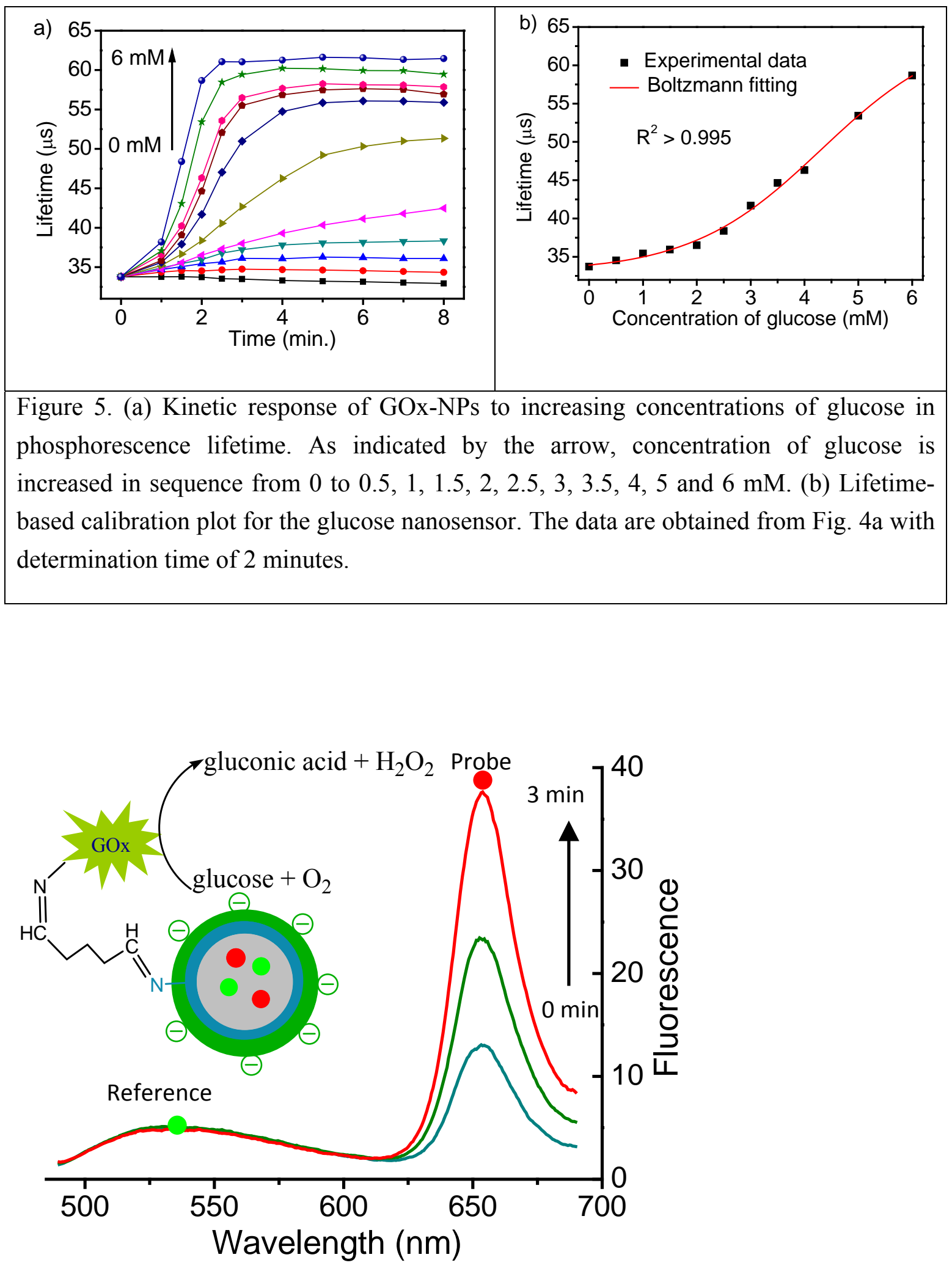